\title{
Transseptal left heart catheterisation guided by intracardiac echocardiography
}

\author{
T Szili-Torok, GP Kimman, D Theuns, J Res, J R T C Roelandt, L J Jordaens
}

\begin{abstract}
Objective-To develop a novel approach of transseptal puncture guided by intracardiac echocardiography and to assess its efficacy.

Methods-Transcatheter intracardiac echocardiography with a $9 \mathrm{MHz}$ rotating transducer was performed to guide transseptal puncture in 12 patients (mean age 43.1 years, range 31-68) who underwent radiofrequency catheter ablation of left sided accessory pathways. Initially, the echocardiography and transseptal catheters were placed adjacent to each other in the superior vena cava and were withdrawn to the level of the fossa ovalis.

Results-The successful puncture site was associated with visualisation of the fossa ovalis (12 patients, $100 \%$ ) and the aorta (12 patients, 100\%), tenting of the fossa (six patients, 50\%), penetration of the needle visualised by the ultrasound catheter (12 patients, $100 \%$ ), and echocardiographic contrast material applied in the left atrium (12 patients, $100 \%)$. The characteristic jump of the needle onto the fossa ovalis was observed simultaneously with fluoroscopy and intracardiac ultrasound (12 patients, 100\%). All procedures were successful. There were no complications associated with the transseptal procedure.

Conclusions-Intracardiac echocardiography is feasible to guide transseptal puncture. The optimal puncture site can be assessed by simultaneous detection of the characteristic downward jump of the transseptal needle onto the fossa ovalis by intracardiac ultrasound and fluoroscopy. (Heart 2001;86:e11)
\end{abstract}

Keywords: intracardiac echocardiography; transseptal catheterisation

Echocardiography provides potentially useful information for electrophysiological studies and pacemaker implantation by visualisation of important anatomical landmarks and structures. $^{12}$ Transoesophageal echocardiography (TOE) improves visualisation for specific indications. ${ }^{3}$ More recently, intracardiac echocardiography was used to guide radiofrequency catheter ablation for atrial flutter and other supraventricular arrhythmias. ${ }^{4-6}$ Recently there has been renewed interest in transseptal left heart catheterisation caused by the development of left sided radiofrequency catheter ablation. ${ }^{7-10}$ Although conventional left sided accessory pathway ablation is effectively performed with a retrograde approach, severe complications have been reported including damage of the aortic

Accepted 11 July 2001 valve, peripheral arterial thromboembolic events, transient ischaemic attacks, dissection of the aorta, and endocarditis. ${ }^{11-13}$ Some investigators prefer a transseptal approach towards the mitral ring, which gives improved catheter stability and has a comparable success rate. ${ }^{814}$ However, a significant number of acute and potentially lethal complications with transseptal puncture may occur. ${ }^{70}$ The aim of the present study was to establish a novel technique that can minimise the potential risks for acute complications in patients undergoing transseptal puncture.

\section{Methods}

PATIENT POPULATION

Twelve consecutive patients were recruited in this study who underwent left heart catheterisation for ablation of left sided accessory pathways. The mean age of the patients was 43.1 years (range 31-68); eight patients were women. None of the patients had structural heart disease.

ELECTROPHYSYOLOGICAL PROCEDURE

Standard electrophysiological study was undertaken under intravenous sedation with 5-10 mg diazepam. After a subclavian venipuncture, a 5 French decapolar diagnostic catheter (Supreme CS, DAIG Corp, St Jude Medical Inc, Minneapolis, Minnesota, USA) was inserted into the coronary sinus. Three 6 French quadripolar diagnostic catheters (Viking, Bard Electrophysiology, Lowell, Massachusetts, USA) were placed high in the right atrium, across the tricuspid valve to record the His potential and in the right ventricular apex. Bipolar intracardiac electrograms, filtered at a bandpass of $30-500 \mathrm{~Hz}$, were Figure 1 ( $A$, left) Anteroposterior fluoroscopy view of the dilator sheath (double arrows)
and the intracardiac ultrasound catheter at the time of the picture in panel $B$. . $B$, right) View of interatrial septum and fossa ovalis with intracardiac echocardiography. The double arrow is pointing to the dilator sheath and the intracardiac echocardiography probe. The dilator sheath is moving in the direction of the fossa ovalis from the contralateral side of the aortic valve. AoV, aortic valve; $L A$, left atrium; $R A$, right atrium. 


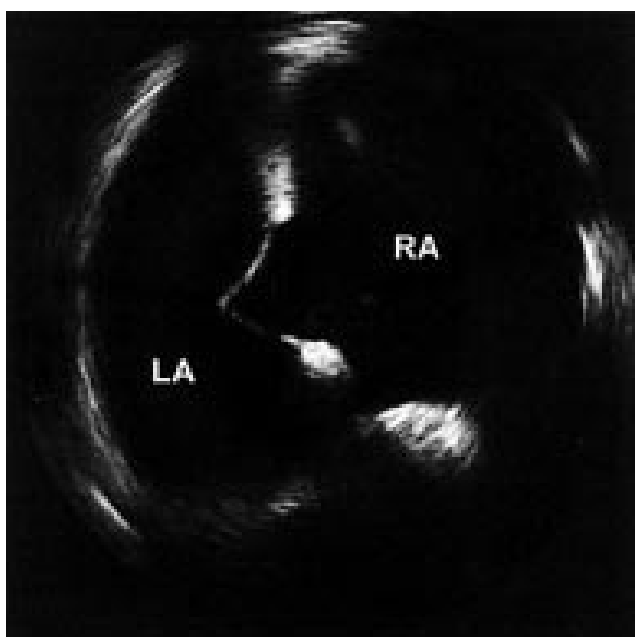

Figure 2 Tenting of the fossa can become very characteristic, as it in this case.

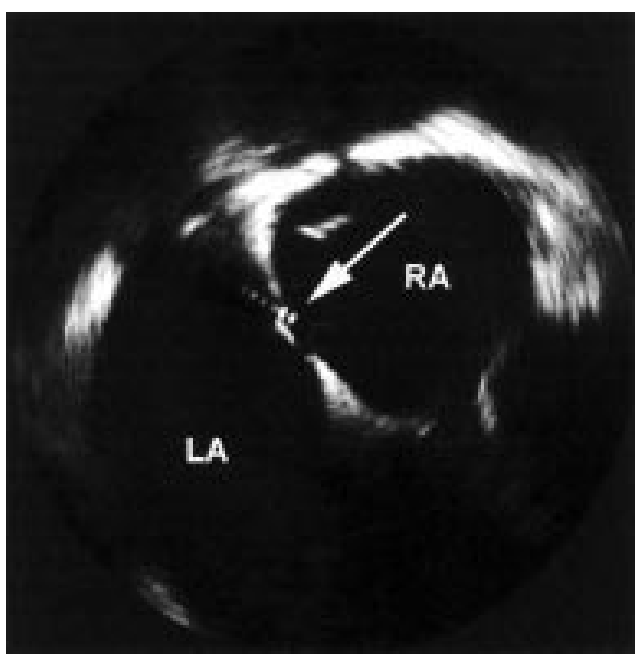

Figure 3 Image of the fossa ovalis after transseptal puncture. The transseptal sheath is penetrating the membrane of the interatrial septum.

recorded simultaneously with a 12 lead ECG (Prucka Engineering, Houston, Texas, USA). Electrophysiological testing was used to confirm the existence of left sided accessory pathways using antegrade and retrograde mapping with atrial and ventricular pacing.

TRANSSEPTAL PUNCTURE

After electrophysiological testing confirmed the existence of a left sided accessory pathway, the left femoral vein was punctured and an 8.5
French multipurpose introducer sheath with a long curve type (model 5662, EP Technologies, Boston Scientific Corp, San Jose, California, USA) was introduced into the superior vena cava (SVC). A $9 \mathrm{MHz}$ rotating, intracardiac ultrasound transducer catheter (model 9900, EP Technologies, Boston Scientific Corp) was filled with $3-5 \mathrm{~cm}^{3}$ sterile water and then connected to an ultrasound console (model I5007, Boston Scientific Corp). The images were displayed on a monitor and recorded on videotape. The ventricular pacing catheter was removed and replaced by a transseptal sheath (SL1, SL2, DAIG Corp), which was introduced to the SVC adjacent to the echocardiography catheter (fig 1A). The transseptal sheath was loaded with a Brockenbrough needle (DAIG Corp), which was advanced to within $1 \mathrm{~cm}$ of the dilator tip. The position was checked in three fluoroscopy views (anteroposterior, left anterior oblique, and right anterior oblique). The intracardiac echocardiography catheter was manually pulled back from the SVC in the direction of inferior vena cava. The pull back was stopped when the fossa ovalis appeared on the screen (fig 1B). The entire transseptal sheath was then withdrawn from the SVC to the right atrium. The movement of the transseptal dilator sheath from the SVC to the level of the fossa ovalis was simultaneously detected by fluoroscopy and intracardiac echocardiography. After a characteristic downward "jump" of the sheath, which is described as an indirect radiological sign of the position in the fossa, the sheath appeared directly in the view of the intracardiac echocardiography catheter. The transseptal sheath was always moving in the direction of the fossa ovalis from the contralateral site of the aortic valve (fig 1B). After the dilator sheath jumped onto the fossa ovalis, confirmed by the intracardiac ultrasound, the septum was approached with the needle (figs 2 and 3). Fluoroscopy and intracardiac echocardiography followed all of the attempts for transseptal puncture simultaneously. Echocardiographic contrast material was injected in to the left atrium and detected by echocardiography to confirm successful puncture. The dilator and the needle were withdrawn and 7000 IU of heparin was administered intravenously. The total procedure time was registered until successful puncture was achieved and confirmed.

Table 1 Results of intracardiac measurements and findings

\begin{tabular}{|c|c|c|c|c|c|c|c|c|c|}
\hline $\begin{array}{l}\text { Patient } \\
\text { number }\end{array}$ & FO size $(\mathrm{mm})$ & $\begin{array}{l}F O-A W \\
\text { distance (mm) }\end{array}$ & Tenting & $\begin{array}{l}\text { TFO- } A W \\
\text { distance (mm) }\end{array}$ & $A o V-F O$ & $\begin{array}{l}\text { AoV-FO } \\
\text { distance (mm) }\end{array}$ & Fump & Needle & $\begin{array}{l}\text { Contrast detection } \\
\text { in } L A\end{array}$ \\
\hline 1 & 4.8 & 15.9 & + & 14.1 & + & 17.9 & + & + & + \\
\hline 2 & 9.2 & 24.2 & + & 15.7 & + & 8.2 & + & + & + \\
\hline 3 & 12.0 & 40.1 & + & 15.6 & - & NA & + & + & + \\
\hline 4 & 9.0 & 40.0 & - & NA & + & 22.1 & + & + & + \\
\hline 5 & 5.6 & 16.0 & - & NA & + & 9.3 & + & + & + \\
\hline 6 & 11.2 & 33.4 & - & NA & - & NA & + & + & + \\
\hline 7 & 6.2 & 42.7 & - & NA & + & 11.6 & + & + & + \\
\hline 8 & 14.4 & 15.6 & + & 10.3 & + & 17.6 & + & + & + \\
\hline 9 & 11.5 & 38.2 & - & NA & - & NA & + & + & + \\
\hline 10 & 8.4 & 26.1 & + & 18.2 & + & 20.2 & + & + & + \\
\hline 11 & 7.2 & 15.0 & + & 8.0 & - & NA & + & + & + \\
\hline 12 & 5.4 & 23.3 & - & NA & + & 32.6 & + & + & + \\
\hline
\end{tabular}

AoV, aortic valve; AW, atrial wall; FO, fossa ovalis; LA, left atrium; NA, not available; TFO, tented fossa ovalis. 


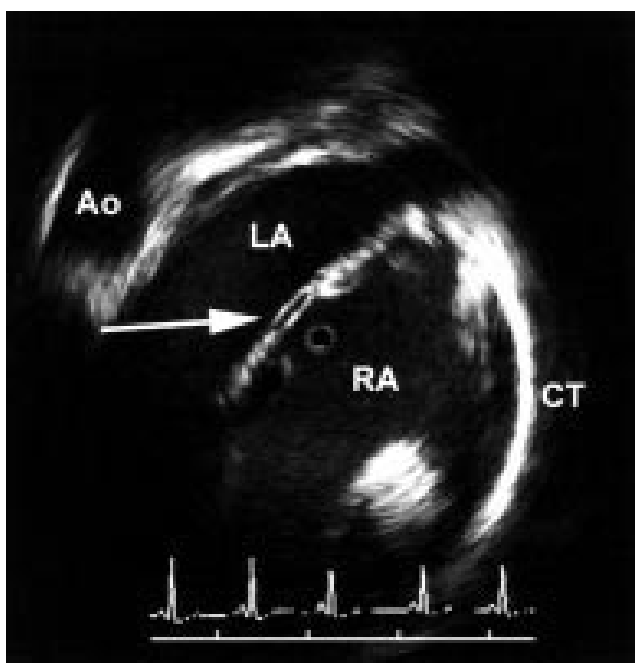

Figure 4 A rare case: a double membrane is clearly visible in the fossa ovalis. Next to the left atrium the aortic arch is visualised. Ao, aorta; CT, crista terminalis.

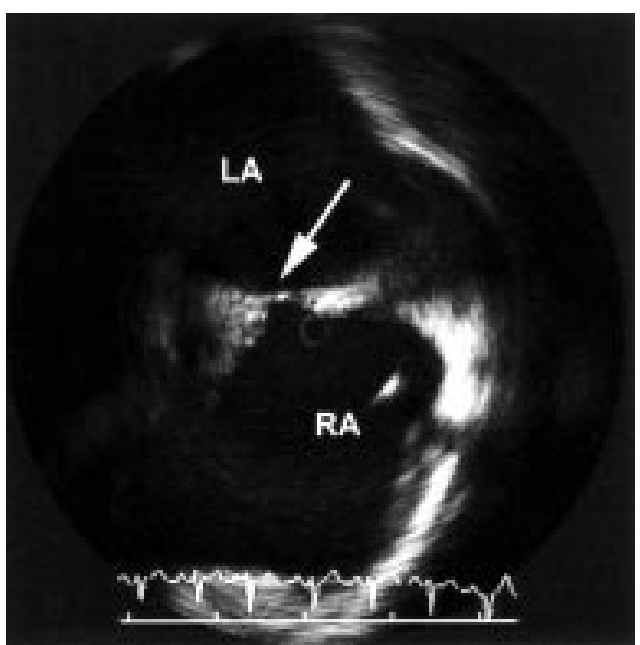

Figure 5 Small fossa ovalis detected by intracardiac echocardiography. In this patient the size of the fossa ovalis was $4.8 \mathrm{~mm}$.

INTRACARDIAC MEASUREMENTS

Intracardiac measurements were taken off line. The size of the fossa ovalis, the distance between the fossa ovalis and left atrial wall, and
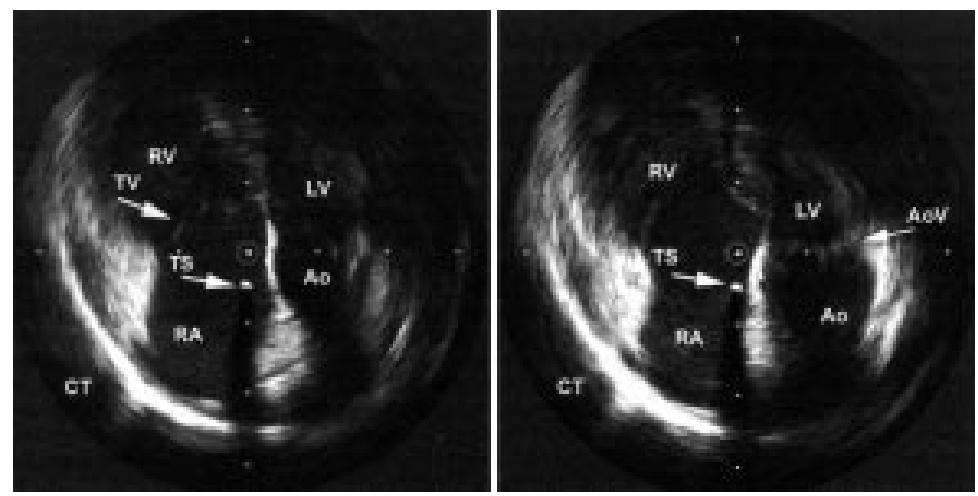

Figure 6 ( $A$, left) The heart at the time of closure of the tricuspid valve. The morphology of the valve in the left side of the heart may be mistaken for the mitral valve; however, the location of the chambers and the asynchronous closure of the valves clearly indicates that this is the aortic valve. (B, right) The same chambers $400 \mathrm{~ms}$ later at the time of closure of the aortic valve. LV, left ventricle; $M V$, mitral valve; $R V$, right ventricle; $T S$, transseptal sheath (the dilator sheath that is pointing towards the membranous right atrial wall adjacent to the aorta at the level of aortic valve). the distance between the tented fossa and left atrial wall were measured.

ABLATION PROCEDURE

After successful transseptal puncture, a standard ablation procedure was undertaken. A 7 French quadripolar mapping/ablation catheter (Model Celsius, type B, D, Biosense Webster Inc, Diamond Bar, California, USA) was used to map the mitral annulus and to deliver radiofrequency energy. Radiofrequency energy was applied to the atrial side of the mitral annulus with continuous atrial and ventricular signals during atrial pacing or during sinus rhythm. The successful pulses resulted in an abrupt loss of accessory pathway conduction. After ablation, ventricular pacing and the administration of intravenous adenosine (12 mg) were used to assess the loss of accessory pathway conduction.

\section{STATISTICAL ANALYSIS}

Continuous variables are expressed as mean (SD). Non-parametric data were compared using Wilcoxon $t$ test. Correlation analysis was performed using the Pearson test. The level of significance was set at $\mathrm{p}=0.05$.

\section{Results}

The mean duration of transseptal puncture was 17.4 (7.9) minutes (range 7.5-38 minutes). Intracardiac echocardiography visualised the following anatomical structures in all cases (12 patients, $100 \%$, table 1): SVC, crista terminalis, and aortic valve. The pulmonary veins were visualised in only one case $(8 \%)$. The view of the fossa ovalis was optimal in all patients. The fossa ovalis, the tip of the needle, the aortic valve, and the left atrial wall were visualised in a single ultrasound image in eight patients $(66 \%)$ (fig 1B). In one patient a double membrane was detected in the fossa ovalis (fig 4). The image of the membrane of the fossa ovalis was stable throughout the procedure in 10 cases. In two cases minor adjustments of the ultrasound catheter were necessary to optimise the image during the procedure. The size of the visualised fossa ovalis was 8.7 (2.9) $\mathrm{mm}$ (range $4.8-14.4 \mathrm{~mm}$ ). There was no correlation of the size of the fossa ovalis and the time to successful puncture (NS, $r=0.11$ ). The distance between the fossa ovalis and the left atrial wall was $27.5(10.8) \mathrm{mm}$ (range 15-42.7 $\mathrm{mm}$ ).

Movement of the transseptal sheath was visible in every case in real time. The characteristic downward jump of the catheter on to the membrane of the fossa ovalis was detected in every patient (12 patients, 100\%). After the jump, the needle was visible in the fossa in every case (12 patients, 100\%) (fig 5). Ultrasound showed the characteristic tenting of the fossa in six cases (50\%) (fig 4). In the cases where the needle tented the fossa ovalis, the mean distance between the peak point of the fossa ovalis and the left atrial wall decreased from $27.5(10.8) \mathrm{mm}$ (range $15-42.7 \mathrm{~mm}$ ) to 13.6 (3.7) $\mathrm{mm}$ (range $8.1-18.2 \mathrm{~mm}$ ) $(\mathrm{p}<0.05)$. The tented fossa did not abut on the left atrial wall in any of our cases. The distance between the needle before approaching 
the membrane and the aortic valve was 19.8 (6.2) $\mathrm{mm}$ (range $11-26.9 \mathrm{~mm}$ ). The first attempt of transseptal puncture guided by intracardiac ultrasound was successful in 10 patients $(83 \%)$. In one patient, the dilator sheath became dislocated after the septum was approached by the needle. The second attempt was successful. In one case only the third attempt was successful as the dilator sheath jumped twice onto the small thin wall between the right atrium and the aorta after the pull back (fig 6). Echocardiographic contrast injection was detected in the left atrium after every successful puncture (12 patients, 100\%). There were no complications associated with the procedure.

\section{Discussion}

TRANSSEPTAL LEFT HEART CATHETERISATION

Percutaneous puncture of the interatrial septum was introduced for catheterisation of the left heart in $1960 .{ }^{15}$ The technique relied on fluoroscopic landmarks to define anatomical boundaries..$^{15}$ In addition, the movement of the tip of the device from the thicker muscular septum to the thin wall of the fossa ovalis is an important sign that can be detected by experts without the need to puncture the femoral artery. Some investigators introduce additional anatomical landmarks such as a pigtail catheter in the aorta (which requires arterial puncture) or use the His bundle or coronary sinus catheters as a reference. However, transseptal catheterisation remains a difficult procedure, particularly in cases where the atrial anatomy is atypical or the fossa ovalis is small. Recently this technique became a frequently used method for left sided radiofrequency ablation. ${ }^{11}$ Further, some sites in the left side cannot be reached with the retrograde technique. The major advantages of this technique are the stability of the catheter and the lack of peripheral arterial puncture. However, a complication rate between $3 \%$ and $5 \%$ has been reported. Most of the complications occur in the periprocedural period. A review of 1279 transseptal punctures in a single centre retrospective study found a $90 \%$ overall success rate, with $1.2 \%$ life threatening complications, including pericardial tamponade, systemic emboli, and death secondary to aortic perforation. ${ }^{10}$ Another centre reported a $91 \%$ success rate with a slightly higher complication rate. Aortic puncture $(0.7 \%)$, pericardial tamponade $(3.2 \%)$, and systemic embolisation $(1.1 \%)$ were the major complications. ${ }^{7}$

IMAGING TECHNIQUES GUIDING TRANSSEPTAL PUNCTURE

Imaging techniques such as TOE and two dimensional transthoracic echocardiography have been used for the assessment of the interatrial septum during transseptal puncture. ${ }^{216-19}$ Both techniques have significant limitations. Transthoracic ultrasound may not be able to locate accurately the thin wall of the fossa ovalis. Furthermore, transthoracic echocardiography is fairly uncomfortable to perform without a risk of violation of sterility during the intervention. TOE was found to be feasible, but cardiac perforation was reported because of inadequate localisation of the fossa ovalis. ${ }^{18}$ Furthermore, it carries the risk of oesophageal bleeding ${ }^{18}$ and limits communication with the patient during the procedure. TOE may cause lengthening of the procedure because of the introduction of the oesophageal probe and requires intravenous sedation for a longer period.

INTRACARDIAC ULTRASOUND FOR TRANSSEPTAL PUNCTURE

Intracardiac ultrasound gives a view of the fossa ovalis with $100 \%$ accuracy. ${ }^{50-22}$ This is a direct method and a possibility to avoid complications. In a recent study 53 patient underwent transseptal puncture by the guidance of intracardiac echocardiography. ${ }^{23}$ The success rate of the first attempt was $96 \%$ and there were no complications associated with the procedure. ${ }^{23}$ In previous studies, identification of the dilator tip was difficult. ${ }^{24}$ In our study we confirmed a successful puncture by using contrast injection through the Brockenbrough needle. The appearance of contrast in the left atrium is a direct sign of successful puncture.

\section{METHODOLOGICAL DIFFERENCES}

In contrast with previous findings, in this study tenting of the membranous interatrial septum was detected in only one half of the patients. This is because of the differences in approaching the septum. After localisation of the transseptal sheath, we approached the septum directly with the needle. In the study of Daoud and colleagues ${ }^{23}$ the dilator was used to cause tenting and the needle was forwarded after the sign of tenting was observed. This resulted in abutting of the fossa on the left atrial wall in a considerable proportion of the patients. ${ }^{23}$ The dilator should then have been readjusted. In our study abutting on the left atrial wall was not detected. We recommend that after the jump, which can be detected simultaneously with fluoroscopy and intracardiac ultrasound, the interatrial septum be approached directly by the needle. This method requires confirmation of the location of the needle in the left atrium. By injection of echocardiographic contrast material this was achieved in all of our patients.

CLINICAL IMPACT

Compared with other imaging techniques intracardiac echocardiography has some significant advantages. The major advantage is excellent visualisation of the fossa ovalis. Furthermore, it provides the possibility of flexible planning of the procedure. Firstly, it does not require deep sedation. Secondly, it can be used comfortably for a longer time. No additional arterial puncture is required.

In conclusion, on the basis of growing experience with intracardiac echocardiography the method seems to be an additional tool to minimise the risk for dangerous complications during the puncture of interatrial septum..$^{13}$ In addition, the most useful indication of the optimal puncture site is the characteristic downward jump of the transseptal needle on to 
the fossa ovalis detected simultaneously with intracardiac ultrasound and fluoroscopy.

1 Lee MS, Evans SJ, Blumberg S, et al. Echocardiographically guided electrophysiologic testing in pregnancy. $7 \mathrm{Am}$ Soc guided electrophysiologic test
Echocardiogr 1994; 7:182-6.

2 Hurrell DG, Nishimura RA, Symanski JD, et al. Echocardiography in the invasive laboratory: utility of two-dimensional echocardiography in performing transseptal catheterization. Mayo Clin Proc 1998;73:126-31.

3 Jordaens LJ, Vandenbogaerde JF, Van de Bruaene P, et al. Transesophageal echocardiography for insertion of a physiological pacemaker in early pregnancy. Pacing Clin Electrophysiol 1990;13:955-7.

4 Olgin JE, Kalman JM, Fitzpatrick AP, et al. Role of right atrial endocardial structures as barriers to conduction during human type I atrial flutter: activation and entrainment mapping guided by intracardiac echocardiography. Circulation 1995;92:1839-48.

5 Lesh MD, Kalman JM, Karch MR. Use of intracardiac echocardiography during electrophysiologic evaluation and therapy of atrial arrhythmias. $\mathcal{F}$ Cardiovasc Electrophysiol 1998;9(8 suppl):S40-7.

6 Kalman JM, Lee RJ, Fisher WG, et al. Radiofrequency catheter modification of sinus pacemaker function guided by eter modification of sinus pacemaker function guided by intracar

7 Blomstrom-Lundqvist C, Olsson SB, Varnauskas E. Transseptal left heart catheterization: a review of 278 studies. Clin Cardiol 1986;9:21-6.

8 Lesh MD, Van Hare GF, Scheinman MM, et al. Comparison of the retrograde and transseptal methods for ablation of left free wall accessory pathways. $7 \mathrm{Am}$ Coll Cardiol 1993;22:542-9

9 Montenero AS, Crea F, Bendini MG, et al. Catheter ablation of left accessory atrioventricular connections: the transseptal approach. F Interv Cardiol 1995;8(6 suppl): 806-12.

10 Roelke M, Smith AJ, Palacios IF. The technique and safety of transseptal left heart catheterization: the Massachusetts General Hospital experience with 1,279 procedures. Cathet Cardiovasc Diagn 1994;32:332-9.

11 Scheinman MM, Huang S. The 1998 NASPE prospective catheter ablation registry. Pacing Clin Electrophysiol 2000; catheter ab:1020-8.

12 Zhou L, Keane D, Reed G, et al. Thromboembolic complications of cardiac radiofrequency catheter ablation: a review of the reported incidence, pathogenesis and current research directions. $f$ Cardiovasc Electrophysiol 1999;10: 611-20.

13 Talwar KK, Singh B, Goel P, et al. In-hospital results of radiofrequency ablation of supraventricular tachycardia. Indian Heart $\mathcal{f}$ 1996;48:685-90.

14 Deshpande SS, Bremner S, Sra JS, et al. Ablation of left free-wall accessory pathways using radiofrequency energy at the atrial insertion site: transseptal versus transaortic approach. F Cardiovasc Electrophysiol 1994;5:219-31.

15 Brockenbrough E, Braunwald E. A new technique for left ventricular angiography and transseptal left heart catheterization. Am f Cardiol 1960;6:1062-7.

16 Hahn K, Gal R, Sarnoski J, et al. Transesophageal echocardiographically guided atrial transseptal catheterization in
patients with normal-sized atria: incidence of complicapatients with normal-sized atria: i
tions. Clin Cardiol 1995;18:217-20.

17 Kantoch MJ, Frost GF, Robertson MA. Use of transesophageal echocardiography in radiofrequency catheter ablation in children and adolescents. Can f Cardiol 1998;14:519-23.

18 Tucker KJ, Curtis AB, Murphy J, et al. Transesophageal echocardiographic guidance of transseptal left heart catheterization during radiofrequency ablation of left-sided accessory pathways in humans. Pacing Clin Electrophysiol 1996;19:272-81.

19 Kyo S, Motoyama T, Miyamoto N, et al. Percutaneous introduction of left atrial cannula for left heart bypass: utility of biplane transesophageal echocardiographic guidance ity of biplane transesophageal echocardiographic guida
for transseptal puncture. Artif Organs 1992;16:386-91.

20 Hung JS, Fu M, Yeh KH, et al. Usefulness of intracardiac echocardiography in transseptal puncture during percutaneous transvenous mitral commissurotomy. Am $\mathcal{F}$ Cardiol 1993;72:853-4

21 Hung JS, Fu M, Yeh $\mathrm{KH}$, et al. Usefulness of intracardiac echocardiography in complex transseptal catheterization during percutaneous transvenous mitral commissurotomy. Mayo Clin Proc 1996;71:134-40.

22 Epstein LM, Smith T, TenHoff H. Nonfluoroscopic transseptal catheterization: safety and efficacy of intracardiac echocardiographic guidance. 7 Cardiovasc Electrophysiol 1998;9:625-30.

23 Daoud EG, Kalbfleisch SJ, Hummel JD. Intracardiac echocardiography to guide transseptal left heart catheterization for radiofrequency catheter ablation. $\mathcal{F}$ Cardiovasc Electrophysiol 1999;10:358-63.

24 Mitchel JF, Gillam LD, Sanzobrino BW, et al. Intracardiac ultrasound imaging during transseptal catheterization. Chest 1995;108:104-8. 